\title{
Tradução e validação de um questionário de avaliação de qualidade de vida em AIDS no Brasil ${ }^{1}$
}

\author{
Patrícia Coelho de Soárez,2 Adauto Castelo, ${ }^{3}$ Paulo Abrão, ${ }^{3}$ \\ William C. Holmes ${ }^{4}$ e Rozana Mesquita Ciconelli ${ }^{2,3}$
}

Como citar de Soárez PC, Castelo A, Abrão P, Holmes WC, Ciconelli RM. Tradução e validação de um questionário de avaliação de qualidade de vida em AIDS no Brasil. Rev Panam Salud Publica. 2009;25(1):69-76.

RESUMO Objetivo. Traduzir para português brasileiro, adaptar culturalmente e avaliar as propriedades psicométricas (validade e confiabilidade) de um questionário de qualidade de vida (HIV / AIDS-Targeted Quality of Life Instrument, HAT-QoL) especifico para pacientes infectados pelo HIV.

Método. Estudo transversal realizado no ambulatório de infectologia da Escola Paulista de Medicina da Universidade Federal de São Paulo. Coletaram-se características sociodemográficas e clínicas de 106 indivíduos infectados pelo HIV, que responderam os questionários de qualidade de vida HAT-QoL e SF-36. O coeficiente de correlação de Pearson aferiu a validade de construto. Para avaliar a confiabilidade foram calculados o alfa de Cronbach e o coeficiente de correlação intraclasse.

Resultados. A proporção de homens na amostra foi de 70,8\%. A média de idade foi 39,9 anos. Dos participantes, $40,5 \%$ eram homo ou bissexuais. Onze $(10,4 \%)$ pacientes tinham contagem de células CD4 $\leq 200$ células $/ \mathrm{mm}^{3}$. Entre os nove domínios do HAT-QoL, sete (função geral, satisfação com a vida, preocupações com a saúde, preocupações com a medicação, aceitação do HIV, confiança no profissional e função sexual) apresentaram efeito teto substancial. O domínio "função sexual" foi o que apresentou efeito teto mais alto $(63,2 \%)$. O domínio preocupações financeiras apresentou efeito chão substancial (30,2\%). Associações estatisticamente significativas foram observadas entre os dominios do HAT-QoL e características sociodemográficas e clínicas e domínios do questionário SF-36. A consistência interna foi satisfatória (alfa de Cronbach $=0,73$ a 0,90). A reprodutibilidade inter e intraobservador foi muito alta $(0,87$ a 0,98 e 0,82 a 0,97 , respectivamente $)$.

Conclusão. O HAT-QoL em português brasileiro é um instrumento válido, confiável e que pode contribuir para avaliar o impacto da infecção pelo HIV sobre a qualidade de vida de pacientes brasileiros.

Palavras-chave Confiabilidade e validade, estudos de validação, qualidade de vida, Síndrome de Imunodeficiência Adquirida, Brasil.

\footnotetext{
1 A versão em português brasileiro do Instrumento HAT-QoL e do Manual de Pontuação estão disponíveis gratuitamente no site da Revista Panamericana de Salud Publica/Pan American Journal of Public Health (http:/ /journal.paho.org), "Online Extras".

2 Universidade Federal de São Paulo (UNIFESP), Centro Paulista de Economia da Saúde. Correspondência: Rua Botucatu 685, CEP 04023-062, São Paulo, SP, Brasil. E-mail: patricia.soarez@cpes. org.br
}

A partir da década de 1990, observouse um notável declínio no número de

Universidade Federal de São Paulo (UNIFESP), Departamento de Medicina.

4 University of Pennsylvania School of Medicine, Center For Health Equity Research and Promotion and Center for Clinical Epidemiology and Biostatistics, Filadélfia, PA, Estados Unidos da América. mortes relacionadas à infecção pelo HIV , atribuído principalmente ao uso da terapia antirretroviral (TARV). A efetividade da TARV em controlar a replicação viral e reduzir a morbimortalidade relacionada à infecção pelo HIV tem sido amplamente demonstrada (1-3). Porém, a cura ainda não é possível; o tratamento é 
prolongado e associa-se a efeitos adversos variados, cujo impacto na qualidade de vida dos pacientes permanece incerto. Atualmente, há 23 drogas antirretrovirais disponíveis, com inúmeras possibilidades de esquemas TARV. Uma vez que a eficácia da maioria desses esquemas é semelhante, a escolha do esquema deverá levar em conta a repercussão na qualidade de vida. Com essa motivação, nos últimos anos notou-se interesse crescente pela mensuração da qualidade de vida relacionada à saúde (QVRS) em indivíduos infectados pelo HIV. A QVRS se refere à percepção do indivíduo sobre o seu funcionamento ou bem estar nas várias dimensões (física, mental, social, etc.) da sua vida (4).

Atualmente, existem diversos instrumentos específicos para avaliar a qualidade de vida na AIDS: Medical Outcomes Questionnaire adaptado para HIV (questionário de desfechos médicos, MOSHIV) (5), AIDS Health Assessment Questionnaire (questionário de avaliação de saúde, AIDS-HAQ) (6), HIV-QOL Questionnaire (questionário de qualidade de vida, HIV-QL31) (6), General Health SelfAssessment (auto-avaliação da saúde geral, GHSA) (7), The Miami Pediatric Quality of Life Questionnaire: Parent Scale (escala paterna do questionário pediátrico de qualidade de vida de Miami, MPQoL) (7), HIV Symptom Index (índice de sintomas do HIV, HIV-SI) (7), Living with HIV (convivendo com o HIV, LWH) (8), Multi-Dimensional Quality of Life Questionnaire for HIV/AIDS (questionário multi-dimensional de qualidade de vida para HIV/AIDS, MQoL-HIV) (9), HIV Overview of Problems - Evaluation System (sistema de avaliação - panorama de problemas do HIV, HOPES) (10, 11), Functional Assessment of HIV Infection (avaliação funcional da infecção por HIV, FAHI) $(10,11)$ e (HIV/AIDS-Targeted Quality of Life (qualidade de vida focada no HIV/AIDS, HAT-QoL) (12-14). Todos esses instrumentos foram criados em língua inglesa e testados inicialmente em países desenvolvidos. Entre esses questionários, o único cujos domínios foram construídos totalmente a partir de sugestões de pacientes com HIV/AIDS foi o HAT-QoL. É, portanto, um questionário de qualidade de vida específico para esses pacientes, garantindo a inclusão de domínios importantes e relevantes.

No Brasil, a QVRS dos pacientes com HIV/AIDS tem sido pouco estudada. Portanto, existe demanda por um instru- mento válido e confiável, que possa ser aplicado localmente. O objetivo do presente trabalho foi traduzir para português brasileiro, adaptar culturalmente e testar as propriedades psicométricas (validade e confiabilidade) do HAT-QoL traduzido.

\section{MATERIAIS E MÉTODOS}

O presente estudo transversal incluiu uma população de pacientes com HIV/ AIDS assistidos no ambulatório de infectologia da Escola Paulista de Medicina da Universidade Federal de São Paulo (UNIFESP), no Brasil, no período de maio a junho de 2007. Eram elegíveis para o estudo os homens e as mulheres com diagnóstico confirmado de infecção pelo HIV, idade $\geq 18$ anos, capazes de se comunicar em português brasileiro, sem qualquer déficit cognitivo e que aceitaram participar do estudo após leitura do termo de consentimento livre e esclarecido. Os pacientes também autorizaram a coleta de dados em seus prontuários. Nessa amostra de conveniência, os indivíduos foram convidados a participar do estudo de forma voluntária e não receberam incentivos financeiros. $\mathrm{O}$ estudo foi aprovado pelo Comitê de Ética em Pesquisa da UNIFESP.

Apesar de o protocolo não oferecer risco imediato aos sujeitos do estudo, considerou-se a possibilidade de um risco subjetivo, pois algumas perguntas remetem à auto-avaliação de comportamento, história de vida e infecção pelo HIV. O possível risco subjetivo se refere ao que a lembrança da história de vida desses pacientes e a consequente infecção pelo HIV poderia causar. É importante ressaltar que, sempre que necessário, deve-se oferecer apoio psicológico a pacientes vulneráveis não apenas por sua condição clínica, mas também por outros fatores, como condição socioeconômica e ausência de suporte social. No presente estudo não foi oferecido apoio psicológico porque nenhum dos pacientes se mostrou psicologicamente vulnerável.

\section{Instrumentos de medida}

Todos os sujeitos responderam os seguintes instrumentos: HAT-QoL, com 34 itens, Medical Outcomes Study 36-Item Short-Form Health Survey (questionário de coleta de dados de saúde em versão curta, SF-36), com 36 itens, e um questionário com 12 itens que avaliava características sociodemográficas, comportamento de risco para o HIV e características de gravidade da doença pelo HIV.

Os 34 itens do HAT-QoL avaliam nove domínios de qualidade de vida: função geral (seis itens), satisfação com a vida (quatro itens), preocupações com a saúde (quatro itens), preocupações financeiras (três itens), preocupações com a medicação (cinco itens), aceitação do HIV (dois itens), preocupações com o sigilo (cinco itens), confiança no profissional (médico, enfermeiro, ou qualquer profissional de saúde que atenda o paciente) (três itens) e função sexual (dois itens). Para responder cada questão, o indivíduo é conduzido a pensar sobre a sua qualidade de vida nas últimas 4 semanas. As respostas têm formato de escala do tipo Likert de cinco pontos: "todo o tempo", "a maior parte do tempo", "parte do tempo", "pouco tempo" e "nunca". Em cada domínio, zero é o escore mais baixo e 100 o melhor escore possível. Quanto maior o escore, menor o impacto da infecção pelo HIV na qualidade de vida dos indivíduos. Em outras palavras, quanto menor o escore, mais acometida a função, maior a preocupação e menor a satisfação com a vida.

Como não existia um questionário de qualidade de vida específico para AIDS já validado no Brasil e que pudesse servir como padrão ouro de comparação, optamos por comparar o HAT-QoL ao SF-36. O SF-36 é o questionário genérico de qualidade de vida mais usado no mundo (15). Suas propriedades psicométricas se mostraram excelentes em diversas doenças. Em estudos de HIV/AIDS, demonstrou boa consistência interna, confiabilidade e validade de construto. Apresentou responsividade à terapia antirretroviral e mudanças nas contagens de linfócitos T e CD4+, carga viral, magnitude e número de sintomas (11).

O SF-36 avalia oito domínios: capacidade funcional (10 itens), aspectos físicos (sete itens), sociais (dois itens), emocionais (cinco itens), dor (dois itens), estado geral de saúde (cinco itens), vitalidade (quatro itens) e saúde mental (um item). Como no caso do HAT-QoL, neste questionário o indivíduo também é levado a refletir sobre a sua qualidade de vida nas últimas 4 semanas. $\mathrm{O}$ escore para cada domínio varia de zero a 100 , sendo zero o pior e 100 o melhor estado de saúde. A partir dos oito domínios do SF-36, são construídos os componentes físico e mental sumarizados.

As características sociodemográficas (como idade, sexo, escolaridade, renda) e 
algumas características clínicas (como tempo de diagnóstico do HIV, orientação sexual, comportamento de risco) foram informadas pelo paciente. Outras variáveis clínicas (contagem de CD4 e estado clínico) foram pesquisadas nos prontuários desses indivíduos. Todos os questionários foram aplicados na forma de entrevista por dois alunos de pós-graduação previamente treinados.

\section{Processo de tradução e adaptação cultural do instrumento}

A tradução e a adaptação cultural do instrumento foram feitas com base nas orientações de normas previamente estabelecidas $(16,17)$, que recomendam tradução, retrotradução (back-translation), revisão por painel de especialistas e adaptação cultural. Antes do início do processo, um dos autores do instrumento original forneceu uma autorização, por escrito, para o desenvolvimento da adaptação brasileira do questionário e se dispôs a colaborar diretamente no processo de tradução e retrotradução (decentring technique), sugerindo a repetição deste processo em alguns itens do questionário. A versão final do HAT-QoL em português e o guia de pontuação do questionário estão disponíveis no site da Revista Panamericana de Salud Publica/Pan American Journal of Public Health (http:/ /journal. paho.org), "Online Extras".

O processo consistiu de: 1) duas traduções independentes feitas por tradutores cuja língua materna era o português brasileiro. Posteriormente, estes mesmos tradutores realizaram uma revisão conjunta para obter uma versão consensual; 2) painel de especialistas, formado por dois pesquisadores de qualidade de vida relacionada à saúde e dois infectologistas, que avaliaram a equivalência entre a versão consensual e a versão original para identificar itens inadequados; 3) retrotradução realizada por tradutor cuja língua materna era o inglês; 4) avaliação da retrotradução e versão original pelo painel de especialistas em colaboração com o autor do questionário e identificação de itens de compreensão duvidosa, com tradução desses itens por um terceiro tradutor; 5) retrotradução realizada por um segundo tradutor cuja língua materna era o inglês; 6) avaliação da segunda retrotradução e versão original pelo painel de especialistas em colaboração com o autor do questionário e identificação de itens de compreensão du- vidosa, com tradução desses itens por um terceiro tradutor; e 7) aplicação da versão consensual em um grupo de 10 pacientes, que foram estimulados a explicar as questões em voz alta e a comentar qualquer dificuldade de compreensão. A versão final do questionário foi aplicada até que $90 \%$ dos itens fossem bem compreendidos por todos os pacientes.

\section{Análises psicométricas: validade de construto e confiabilidade e reprodutibilidade}

A validade de construto reflete a força das relações lógicas esperadas entre os domínios do instrumento e outras medidas de qualidade de vida relacionada à saúde e medidas clínicas. A validade de construto do HAT-QoL foi medida a partir da matriz de correlações entre os domínios do HAT-QoL e características sociodemográficas e clínicas e entre os domínios do HAT-QoL e os domínios do SF36, usando o coeficiente de correlação de Pearson. As correlações foram categorizadas usando a seguinte classificação: itens altamente correlacionados $(\geq 0,75)$, moderadamente correlacionados $(0,51$ a 0,75$)$, pouco correlacionados $(0,26$ a 0,50$)$ e minimamente correlacionados $(\leq 0,25)(18)$.

A validade de construto também foi avaliada usando as variáveis sociodemográficas (sexo, escolaridade, renda) e clínicas (contagem de CD4, incapacidade, etc.). Essas variáveis foram dicotomizadas (por exemplo, um subgrupo de sujeitos com contagem de CD4 $\leq 200$ e um subgrupo com contagem de CD4 > 200) e as médias dos domínios do HAT-QoL de cada subgrupo foram comparadas.

Para avaliar a confiabilidade interobservador, foi realizada uma segunda aplicação do HAT-QoL 30 minutos após a inicial. A confiabilidade intraobservador foi avaliada através de uma terceira aplicação, com intervalo de 7 a 14 dias.

\section{Análises estatísticas}

Para cada domínio dos questionários foram calculados média, mediana, desvio-padrão, mínimo e máximo, efeito chão e efeito teto. $\mathrm{O}$ efeito chão e o efeito teto (porcentagem de sujeitos que tiveram o escore mais baixo - zero - ou mais alto - 100 - em cada domínio, respectivamente) foram considerados substanciais se ultrapassassem 20\% (19).

As médias foram comparadas através do teste $\mathrm{t}$ de Student bidirecional para variáveis contínuas. As variáveis categóricas foram comparadas pelo teste do qui-quadrado $\left(\chi^{2}\right)$ ou teste exato de Fisher. A confiabilidade ou reprodutibilidade foi avaliada através do alfa de Cronbach do coeficiente de correlação intraclasse (CCI). Um alfa de Cronbach e CCI $\geq 0,70$ foram considerados aceitáveis (20).

O nível de significância utilizado foi de 0,05 . Os dados foram armazenados e analisados através dos programas STATA versão 8.0 e SPSS versão 12.0.

\section{RESULTADOS}

A versão final foi bem aceita pelos pacientes e se mostrou compreensível e de fácil e rápida aplicação (10 a 15 minutos). Nenhuma questão foi considerada não aplicável ou necessitou ser modificada, comprovando o sucesso da adaptação cultural do instrumento.

A avaliação das propriedades psicométricas do instrumento incluiu 106 pacientes. A maioria era do sexo masculino (70,8\%); a média de idade foi de 39,9 anos (DP $= \pm 9,4$ anos). Quarenta e três $(40,5 \%)$ se disseram homo ou bissexuais e $11(10,4 \%)$ tinham contagem de células CD4 $\leq 200$ células $/ \mathrm{mm}^{3}$. Em 44 pacientes $(41,5 \%)$, a doença foi classificada de acordo com os critérios dos Centers for Disease Control and Prevention (CDC) como não avançada (CDC A); em 24 $(22,6 \%)$, como doença moderadamente avançada (CDC B); e em 38 (35,9\%), como doença avançada (CDC C) (tabela 1).

A tabela 2 apresenta a descrição dos escores do HAT-QoL. Todos os domínios apresentaram média entre 40,1 e 77,7, com exceção dos domínios preocupações com a medicação e confiança no profissional, que tiveram médias de 82,1 e 85,5 , respectivamente. Os sete domínios restantes apresentaram efeito teto substancial. O domínio função sexual foi o que apresentou o efeito teto mais alto $(63,2 \%)$. Somente o domínio preocupações financeiras apresentou efeito chão substancial $(30,2 \%)$.

A tabela 3 apresenta a descrição dos escores do SF-36. Todos os domínios apresentaram médias entre 66,7 e 84,5. Cinco domínios (capacidade funcional, aspectos físicos, dor, aspectos sociais e aspectos emocionais) apresentaram efeito teto substancial. O domínio aspectos físi$\cos$ foi o que apresentou efeito teto mais alto $(60,4 \%)$. Nenhum domínio apresentou efeito chão substancial. 
TABELA 1. Características sociodemográficas e clínicas da amostra estudada para validação de um questionário de avaliação de qualidade de vida em AIDS no Brasil

\begin{tabular}{|c|c|c|}
\hline Característica & No. & $\%(n=106)$ \\
\hline \multicolumn{3}{|l|}{ Sexo } \\
\hline Masculino & 75 & 70,8 \\
\hline Feminino & 31 & 29,2 \\
\hline Idade média (DP) mínimo e máximo & \multicolumn{2}{|c|}{$39,9(9,4) \quad 20-65$} \\
\hline \multicolumn{3}{|l|}{ Escolaridade } \\
\hline Ensino fundamental incompleto & 22 & 20,8 \\
\hline Ensino fundamental & 24 & 22,7 \\
\hline Ensino médio & 43 & 40,6 \\
\hline Ensino superior & 17 & 16,0 \\
\hline \multicolumn{3}{|l|}{ Situação laboral atual } \\
\hline Aposentado & 14 & 13,2 \\
\hline Desempregado & 20 & 18,9 \\
\hline Afastado do trabalho pelo INSS a por incapacidade em virtude da AIDS & 6 & 5,7 \\
\hline Empregado & 49 & 46,2 \\
\hline Atua no mercado informal & 3 & 2,8 \\
\hline Autônomo & 14 & 13,2 \\
\hline \multicolumn{3}{|l|}{ Renda salarial individual (salários-mínimos)b } \\
\hline$<1$ & 10 & 9,4 \\
\hline 1 a 3 & 60 & 56,6 \\
\hline 4 a 6 & 24 & 22,6 \\
\hline 7 a 9 & 4 & 3,8 \\
\hline 10 a 12 & 3 & 2,8 \\
\hline 13 a 15 & 4 & 3,8 \\
\hline 16 ou mais & 1 & 0,9 \\
\hline \multicolumn{3}{|l|}{ Orientação sexual } \\
\hline Homossexual masculino & 33 & 31,1 \\
\hline Homossexual feminino & 1 & 0,9 \\
\hline Bissexual masculino & 9 & 8,5 \\
\hline Bissexual feminino & - & - \\
\hline Heterossexual masculino & 33 & 31,1 \\
\hline Heterossexual feminino & 30 & 28,3 \\
\hline \multicolumn{3}{|l|}{ Comportamento de risco para HIVc } \\
\hline Uso de drogas injetáveis & 5 & 4,7 \\
\hline Transfusões & 12 & 11,3 \\
\hline Práticas sexuais inseguras & 55 & 51,9 \\
\hline Não relataram comportamento de risco & 37 & 34,9 \\
\hline Tempo de soropositividade $\geq 24$ meses & 78 & 73,6 \\
\hline Tempo de soropositividade médio (DP) mínimo e máximo & \multicolumn{2}{|c|}{$6,9(5,3) \quad 1-23$} \\
\hline \multicolumn{3}{|l|}{ Contagem de CD4 (células $/ \mathrm{mm}^{3}$ ) } \\
\hline$\leq 200$ & 11 & 10,4 \\
\hline$>200$ & 76 & 71,7 \\
\hline Sem exame & 19 & 17,9 \\
\hline Contagem de CD4 (células $/ \mathrm{mm}^{3}$ ) média (DP) mínimo e máximo & \multicolumn{2}{|c|}{$490,1(285,8) 12-1327$} \\
\hline Condição clínica ${ }^{d}$ & & \\
\hline Assintomático (CDC A) & 44 & 41,5 \\
\hline Sintomático (CDC B) & 24 & 22,6 \\
\hline AIDS (CDC C) & 38 & 35,9 \\
\hline Incapacidade em virtude da AIDS & 3 & 2,8 \\
\hline Recebe auxílio em virtude da AIDS & 19 & 17,9 \\
\hline
\end{tabular}

a INSS: Instituto Nacional do Seguro Social.

b Salário mínimo: R\$ 384 (US\$210).

c Alguns indivíduos podem ter múltiplos comportamentos de risco.

${ }^{d}$ Conforme critérios dos Centers for Disease Control and Prevention (CDC). CDC A: doença não avançada; CDC B: doença moderadamente avançada; CDC C: doença avançada.

\section{Avaliação da validade de construto}

As correlações entre os domínios do HAT-QoL e as características sociodemográficas e de gravidade da doença são apresentadas na tabela 4. Associações estatisticamente significativas foram observadas entre características sociodemográficas e seis domínios do HAT-QoL: função geral, preocupações com a saúde, preocupações financeiras, aceitação do HIV, preocupações com o sigilo e função sexual. Porém, as correlações foram baixas, com valor de $r$ variando de 0,19 a 0,33 . Houve associação significativa entre características de gravidade da doença e três domínios do HAT-QoL (satisfação com a vida, preocupações financeiras e aceitação do HIV), apesar dos baixos coeficientes de correlação, que variaram de $r=0,19$ a $r=0,30$.

A tabela 5 apresenta as médias dos escores de cada domínio nos respectivos subgrupos das variáveis sociodemográficas e de severidade da doença. Os escores de alguns domínios do HAT-QoL foram capazes de discriminar os subgrupos. Pacientes do sexo feminino revelaram pior função sexual do que os do sexo masculino. Indivíduos com escolaridade superior a 12 anos de estudo e renda maior do que 7 salários mínimos apresentaram menos preocupações financeiras e melhor aceitação do HIV. Tempo de diagnóstico $\geq 24$ meses se associou com menor preocupação com o sigilo. Os pacientes com incapacidade relacionada ao HIV relataram função geral e sexual muito inferiores, menos satisfação com a vida e maiores preocupações financeiras do que aqueles sem incapacidade. Os subgrupos CDC A, CDC B e CDC C também foram testados, mas os escores dos domínios nesses três subgrupos não mostraram diferença estatisticamente significativa.

A tabela 6 apresenta as correlações entre os domínios do HAT-QoL e os do SF-36. Houve associação estatisticamente significativa entre os domínios do SF-36 e todos os domínios do HAT-QoL, exceto o domínio confiança no profissional. Correlações moderadas e estatisticamente significativas foram observadas entre os domínios do SF-36 e dois domínios do HAT-QoL (vitalidade e função geral).

\section{Avaliação da confiabilidade}

Todos os domínios demonstraram consistência interna satisfatória, com valores de alfa de Cronbach variando de 0,73 a 0,90 . Os coeficientes de correlação intraclasse também ultrapassaram o critério de 0,70 . Na confiabilidade intraobservador (reprodutibilidade), os coeficientes de correlação intraclasse apresentaram valores entre 0,82 e 0,97 , e na avaliação da confiabilidade interobservador apresentaram valores entre $0,87 \mathrm{e}$ 0,98 (tabela 7). Esses resultados demonstram que a versão em português brasileiro do HAT-QoL apresenta confiabilidade excelente.

\section{DISCUSSÃO}

Este estudo traduziu e avaliou as propriedades psicométricas da versão em 
TABELA 2. Escores da versão do HIV/AIDS-Targeted Quality of Life Instrument em português brasileiro

\begin{tabular}{|c|c|c|c|c|c|c|c|}
\hline Escala & Itens & Média & Mediana & $\begin{array}{l}\text { Desvio- } \\
\text { padrão }\end{array}$ & $\begin{array}{l}\text { Mínimo e } \\
\text { máximo }\end{array}$ & $\begin{array}{c}\text { Efeito chão } \\
(\%)^{a}\end{array}$ & $\begin{array}{c}\text { Efeito teto } \\
(\%)^{\mathrm{a}}\end{array}$ \\
\hline Função geral & 6 & 75,8 & 88,0 & 26,4 & 4 a 100 & 0 & 26,4 \\
\hline Satisfação com a vida & 4 & 73,1 & 81,0 & 27,7 & 0 a 100 & 2,8 & 28,3 \\
\hline Preocupações com a saúde & 4 & 75,3 & 88,0 & 27,9 & 0 a 100 & 2,8 & 34,0 \\
\hline Preocupações financeiras & 3 & 42,6 & 33,0 & 39,2 & 0 a 100 & 30,2 & 13,2 \\
\hline Preocupações com a medicação & 5 & 82,1 & 90,0 & 22,7 & 10 a 100 & 0 & 21,7 \\
\hline Aceitação do HIV & 2 & 64,8 & 75,0 & 38,5 & 0 a 100 & 16,0 & 44,3 \\
\hline Preocupação com o sigilo & 5 & 40,1 & 35,0 & 33,7 & 0 a 100 & 17,9 & 8,5 \\
\hline Confiança no profissional & 3 & 85,5 & 100,0 & 23,2 & 0 a 100 & 0,9 & 55,7 \\
\hline Função sexual & 2 & 77,7 & 100,0 & 33,9 & 0 a 100 & 7,5 & 63,2 \\
\hline
\end{tabular}

a $O$ efeito chão indica o percentual de indivíduos que obtiveram escore mínimo no domínio e o efeito teto indica o percentual de indivíduos que obtiveram escore máximo no domínio.

TABELA 3. Escores do Medical Outcomes Study 36-Item Short-Form Health Survey em estudo para validação do HIV/AIDS-Targeted Quality of Life Instrument em português brasileiro

\begin{tabular}{|c|c|c|c|c|c|c|c|}
\hline Escala & Itens & Média & Mediana & $\begin{array}{l}\text { Desvio- } \\
\text { padrão }\end{array}$ & $\begin{array}{l}\text { Mínimo e } \\
\text { máximo }\end{array}$ & $\begin{array}{c}\text { Efeito chão } \\
(\%)^{a}\end{array}$ & $\begin{array}{c}\text { Efeito teto } \\
(\%)^{a}\end{array}$ \\
\hline Capacidade funcional & 10 & 83,3 & 95,0 & 9,4 & 20 a 65 & 0,9 & 45,3 \\
\hline Aspecto físico & 7 & 84,5 & 100,0 & 24,2 & 0 a 100 & 0,9 & 60,4 \\
\hline Dor & 2 & 71,4 & 72,0 & 27,1 & 0 a 100 & 1,9 & 37,7 \\
\hline Estado geral de saúde & 5 & 73,0 & 79,5 & 24,6 & 5 a 100 & - & 9,4 \\
\hline Vitalidade & 4 & 68,0 & 75,0 & 27,3 & 0 a 100 & 2,8 & 12,3 \\
\hline Aspectos sociais & 1 & 75,0 & 75 & 27,4 & 0 a 100 & 2,8 & 41,5 \\
\hline Aspectos emocionais & 5 & 82,1 & 100,0 & 28,7 & 0 a 100 & 3,8 & 61,3 \\
\hline Saúde mental & 1 & 66,7 & 75,0 & 27,7 & 0 a 100 & 4,7 & 11,3 \\
\hline Componente físico sumarizado ${ }^{b}$ & NA & 50,7 & 52,6 & 8,6 & 19 a 62 & NA & NA \\
\hline Componente mental sumarizado & NA & 47,8 & 51,5 & 12,8 & 10 a 66 & NA & NA \\
\hline
\end{tabular}

a $O$ efeito chão indica o percentual de indivíduos que obtiveram escore mínimo no domínio e o efeito teto indica o percentual de indivíduos que obtiveram escore máximo no domínio.

${ }^{\mathrm{b}} \mathrm{NA}=$ não se aplica.

TABELA 4. Correlações de Pearson entre as escalas do HIV/AIDS-Targeted Quality of Life Instrument em português brasileiro e as características sociodemográficas e de gravidade da doença

\begin{tabular}{|c|c|c|c|c|c|c|c|c|c|}
\hline \multirow[b]{2}{*}{ Característica } & \multicolumn{9}{|c|}{ HIV/AIDS-Targeted Quality of Life Instrument ${ }^{\mathrm{a}}$} \\
\hline & $\mathrm{FG}$ & SV & PS & PF & PM & AHIV & PSi & $\mathrm{CP}$ & FS \\
\hline Idade & $-0,08$ & 0,05 & $0,20^{b}$ & $-0,01$ & 0,03 & 0,07 & 0,13 & $-0,05$ & $-0,16$ \\
\hline Sexo & $-0,13$ & 0,02 & 0,07 & $-0,17$ & 0,00 & $-0,02$ & 0,02 & 0,03 & $-0,25$ \\
\hline Educação & 0,10 & 0,05 & 0,11 & $0,33^{c}$ & 0,18 & $0,27^{c}$ & $0,20^{\mathrm{b}}$ & $-0,17$ & $0,22^{b}$ \\
\hline Situação laboral atual & $0,26^{c}$ & 0,17 & 0,03 & 0,06 & 0,07 & $-0,04$ & $-0,16$ & 0,01 & $0,33^{c}$ \\
\hline Renda salarial individual & 0,12 & 0,14 & 0,00 & $0,29^{c}$ & 0,15 & $0,22^{c}$ & $-0,01$ & $-0,05$ & $0,23^{c}$ \\
\hline Recebe auxílio em virtude da AIDS & $-0,23^{b}$ & $-0,05$ & $-0,10$ & $-0,13$ & $-0,08$ & 0,06 & 0,06 & 0,02 & $-0,05$ \\
\hline Orientação sexual & 0,00 & 0,12 & 0,17 & $-0,30^{c}$ & 0,09 & $-0,01$ & 0,14 & 0,05 & $-0,10$ \\
\hline Uso de drogas injetáveis & $-0,08$ & 0,10 & 0,07 & $-0,03$ & 0,16 & 0,10 & 0,01 & $-0,06$ & $-0,06$ \\
\hline Transfusões & $-0,07$ & $-0,01$ & 0,06 & $-0,11$ & $-0,05$ & 0,08 & 0,11 & 0,08 & 0,07 \\
\hline Práticas sexuais inseguras & $-0,07$ & $-0,19^{b}$ & $-0,16$ & 0,16 & $-0,01$ & $-0,01$ & 0,00 & $-0,11$ & 0,08 \\
\hline Tempo de soropositividade & $-0,05$ & 0,00 & 0,13 & $-0,11$ & $-0,06$ & $0,19 b$ & 0,15 & 0,04 & $-0,11$ \\
\hline Contagem de CD4 (células $/ \mathrm{mm}^{3}$ ) & $-0,06$ & $-0,17$ & 0,16 & $-0,00$ & 0,08 & 0,16 & $-0,00$ & $-0,10$ & $-0,09$ \\
\hline Condição clínica & $-0,07$ & $-0,13$ & $-0,16$ & $-0,08$ & $-0,09$ & $-0,09$ & $-0,01$ & $-0,05$ & 0,09 \\
\hline Incapacidade em virtude da AIDS & $-0,37^{c}$ & $-0,36^{c}$ & $-0,28^{c}$ & $-0,18$ & $-0,36^{c}$ & $-0,14$ & $-0,12$ & $-0,07$ & $-0,35^{c}$ \\
\hline
\end{tabular}

a FG = função geral; SV = satisfação com a vida; PS = preocupações com a saúde; PF = preocupações financeiras; PM = preocupações com a medicação; $A$ HIV = aceitação do HIV; PSi = preocupações com o sigilo; $\mathrm{CP}=$ confiança no profissional; $\mathrm{FS}=$ função sexual. Altamente correlacionados: $\geq 0,75$; moderadamente correlacionados: 0,51 a 0,75 ; pouco correlacionados: 0,26 a 0,50 ; minimamente correlacionados: $\leq 0,25$.

${ }^{b} P<0,05$.

$c P<0,01$.

português do Brasil do HAT-QoL. Embora esse instrumento já tivesse sido aplicado anteriormente em uma população feminina com HIV / AIDS no Brasil
(21), suas propriedades psicométricas não haviam sido testadas.

No presente estudo, o instrumento mostrou bom desempenho. É interessante notar que, embora o HAT-QoL tenha sido originalmente desenvolvido a partir das sugestões de indivíduos soropositivos e portanto deveria apresentar domínios 
TABELA 5. Avaliação da validade de construto usando características sociodemográficas e de gravidade da doença, estudo sobre validação do HIV/AIDS-Targeted Quality of Life Instrument no Brasil

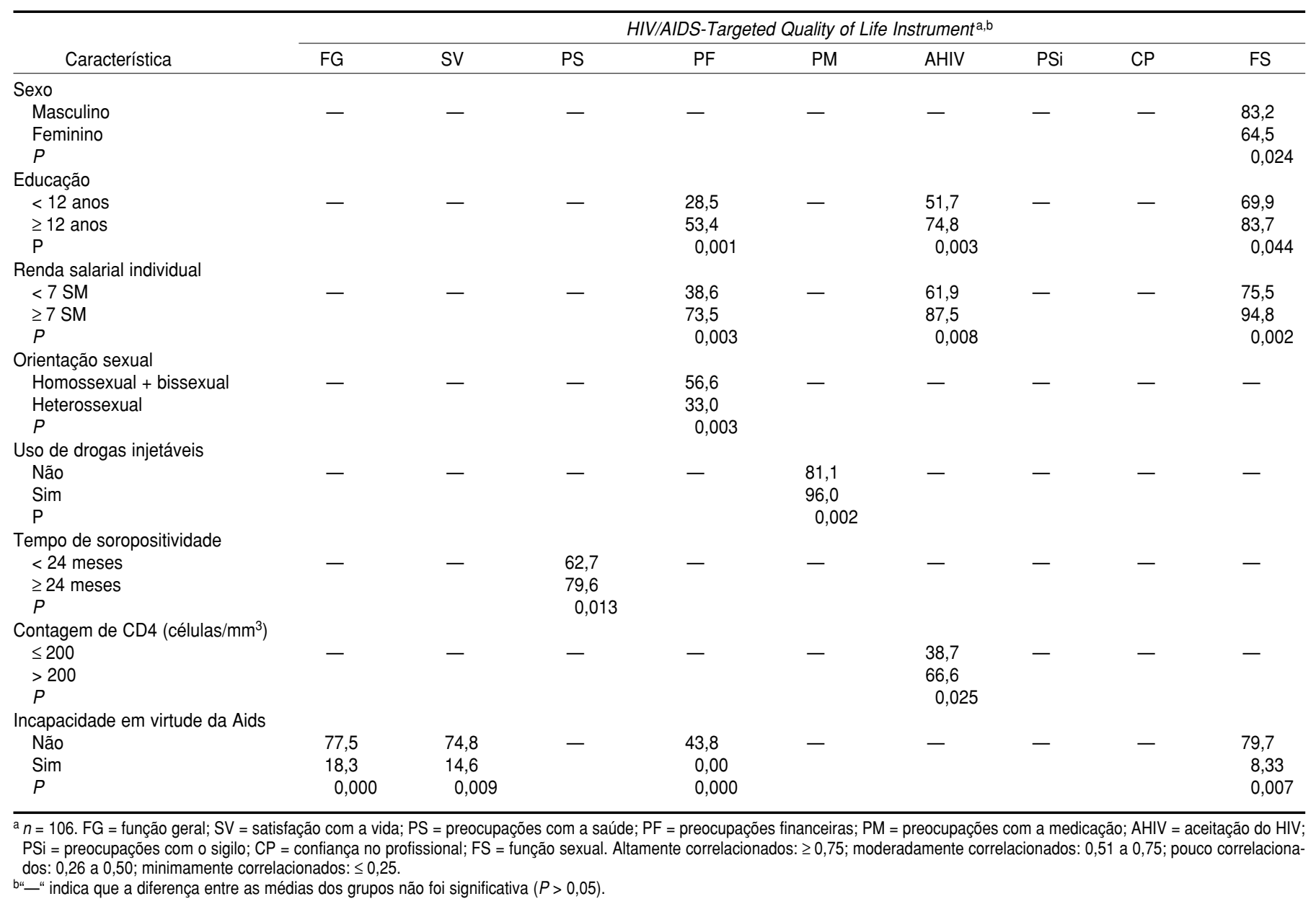

TABELA 6. Correlações de Pearson entre os escores do Outcomes Study 36-Item Short-Form Health Survey (SF-36) e do HIV/AIDS-Targeted Quality of Life Instrument

\begin{tabular}{|c|c|c|c|c|c|c|c|c|c|}
\hline \multirow[b]{2}{*}{ SF-36 } & \multicolumn{9}{|c|}{ HIV/AIDS-Targeted Quality of Life Instrument ${ }^{a}$} \\
\hline & $F G$ & SV & PS & PF & PM & AHIV & PSi & $\mathrm{CP}$ & FS \\
\hline Capacidade funcional & $0,71^{b}$ & $0,62^{b}$ & $0,31^{b}$ & $0,36^{\mathrm{b}}$ & $0,36^{\mathrm{b}}$ & 0,07 & $-0,04$ & 0,05 & $0,25^{b}$ \\
\hline Dor & $0,70^{b}$ & $0,64^{b}$ & $0,33^{b}$ & $0,25^{\mathrm{b}}$ & $0,49^{b}$ & 0,12 & 0,04 & 0,16 & $0,32^{b}$ \\
\hline Estado geral de saúde & $0,65^{\mathrm{b}}$ & $0,68^{\mathrm{b}}$ & $0,58^{\mathrm{b}}$ & $0,27^{b}$ & $0,54^{b}$ & $0,27^{b}$ & 0,10 & 0,07 & $0,24^{c}$ \\
\hline Vitalidade & $0,86^{b}$ & $0,78^{\mathrm{b}}$ & $0,43^{b}$ & $0,42^{b}$ & $0,55^{\mathrm{b}}$ & $0,21^{\mathrm{c}}$ & 0,06 & 0,01 & $0,38^{b}$ \\
\hline Aspectos sociais & $0,69^{b}$ & $0,66^{\mathrm{b}}$ & $0,47^{b}$ & $0,34^{\mathrm{b}}$ & $0,53^{\mathrm{b}}$ & $0,31^{\mathrm{b}}$ & 0,16 & 0,07 & $0,32^{b}$ \\
\hline Componente físico sumarizado & $0,69^{b}$ & $0,60^{\mathrm{b}}$ & $0,36^{\mathrm{b}}$ & $0,25^{b}$ & $0,42^{b}$ & 0,11 & $-0,07$ & 0,10 & $0,22^{\mathrm{c}}$ \\
\hline Componente mental sumarizado & $0,78^{\mathrm{b}}$ & $0,78^{\mathrm{b}}$ & $0,56^{\mathrm{b}}$ & $0,41^{b}$ & $0,63^{b}$ & $0,31^{\mathrm{b}}$ & $0,25^{b}$ & 0,06 & $0,41^{b}$ \\
\hline
\end{tabular}

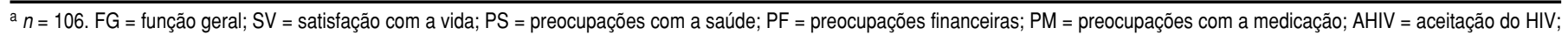
$\mathrm{PSi}$ = preocupações com o sigilo; $\mathrm{CP}=$ confiança no profissional; $\mathrm{FS}=$ função sexual. Altamente correlacionados: $\geq 0,75$; moderadamente correlacionados: 0,51 a 0,$75 ;$ pouco correlacionados: 0,26 a 0,50 ; minimamente correlacionados: $\leq 0,25$.

b $P<0,01$

c $P<0,05$

relevantes para essa população — um efeito teto substancial foi observado em sete dos nove domínios. Avaliações anteriores $(13,14)$ também obtiveram um efeito teto muito alto (44 e 64\%) no domínio confiança no profissional, à semelhança dos $55,7 \%$ do presente estudo. Provavelmente esse resultado sofreu in- fluência do efeito halo: os indivíduos responderam as questões enquanto esperavam por ou imediatamente após o atendimento no ambulatório de infectologia. 
TABELA 7. Confiabilidade da versão brasileira do HIV/AIDS-Targeted Quality of Life Instrument

\begin{tabular}{|c|c|c|c|}
\hline \multirow[b]{2}{*}{ Escala } & \multicolumn{3}{|c|}{ Coeficiente de correlação intraclasse ${ }^{a}$} \\
\hline & $\begin{array}{c}\text { Intra- } \\
\text { observador }\end{array}$ & $\begin{array}{c}\text { Inter- } \\
\text { observador }\end{array}$ & $\begin{array}{c}\text { Alfa de } \\
\text { Cronbach }^{\mathrm{b}}\end{array}$ \\
\hline Função geral & 0,82 & 0,90 & 0,86 \\
\hline Satisfação com a vida & 0,84 & 0,90 & 0,87 \\
\hline Preocupações com a saúde & 0,91 & 0,95 & 0,80 \\
\hline Preocupações financeiras & 0,93 & 0,96 & 0,90 \\
\hline Preocupações com a medicação & 0,90 & 0,96 & 0,73 \\
\hline Aceitação do HIV & 0,97 & 0,98 & 0,80 \\
\hline Preocupação com o sigilo & 0,95 & 0,95 & 0,82 \\
\hline Confiança no profissional & 0,82 & 0,87 & 0,78 \\
\hline Função sexual & 0,94 & 0,97 & 0,87 \\
\hline
\end{tabular}

${ }^{a}$ As análises foram feitas com uma sub-amostra de 31 pacientes estáveis que completaram as três entrevistas.

${ }^{b}$ Consistência interna medida pelo alfa de Cronbach. Análise feita com a amostra de 106 pacientes.

Assim, pode ter havido uma pressão inconsciente para avaliar os profissionais de saúde de uma forma mais positiva. Por outro lado, como constatado anteriormente pelo próprio autor do questionário, esse é um domínio com problemas, que precisa de uma revisão substancial de seus itens para permanecer no questionário.

Neste estudo, o domínio aceitação do HIV ainda apresentou efeito teto superior $(44,3 \%)$ aos $24 \%$ encontrado pelos idealizadores do instrumento (14). O domínio que apresentou efeito teto mais alto $(63,2 \%)$ foi função sexual. Esse resultado não foi encontrado em estudos anteriores, e pode estar refletindo uma amostra local mais saudável e menos comprometida do que as demais. É possível ainda que no contexto local esse construto seja tão valorizado que os indivíduos se avaliaram com nota superior ao estado de saúde real, por não admitirem comprometimento nessa área.

Foi avaliado se o efeito teto variou nas categorizações clínicas CDC A, CDC B e CDC C. Os domínios função sexual, confiança no profissional, aceitação do HIV e preocupações com a medicação apresentaram o mesmo percentual de efeito teto nos pacientes classificados como CDC A e CDC C. No entanto, nos domínios função geral, satisfação com a vida e preocupações com a saúde observou-se maior percentual de efeito teto nos indivíduos CDC A. É provável que esses domínios não apresentem problemas e o efeito teto tenha sido conseqüência do bom estado de saúde desses indivíduos. O problema do efeito teto é que ele limita a responsividade do instrumento. Na prática, os domínios com esse efeito não serão capazes de capturar acréscimos ou decréscimos na qualidade de vida que ocorram acima do mais alto escore da dimensão (12). Fica a dúvida sobre se todos esses domínios realmente apresentaram efeito teto ou se essa amostra apresentava doença controlada e bom estado de saúde.

À semelhança do que foi demonstrado em outro trabalho (22), encontrou-se efeito teto em algumas dimensões do SF36: capacidade funcional, aspectos físicos e emocionais. Além disso, encontrou-se efeito teto nos domínios dor e aspectos sociais. Esses achados corroboram a hipótese de que essa amostra é composta por indivíduos em quem a doença está controlada.

$\mathrm{Na}$ avaliação da validade através das correlações entre os domínios do HATQoL e SF-36, além das correlações estatisticamente significativas com domínios que teoricamente mediam o mesmo construto, um achado interessante que comprovou a validade de construto do instrumento foi a ausência de correlação com domínios que mediam construtos diferentes. Por exemplo, os domínios capacidade funcional, aspecto físico, dor e estado geral de saúde, vitalidade e aspectos sociais do SF-36 não se correlacionaram com os domínios preocupação com o sigilo e confiança no profissional do HAT-QoL.

Os escores de alguns domínios do HAT-QoL foram capazes de discriminar subgrupos com características sociodemográficas e clínicas diferenciadas. Em especial, o grupo de indivíduos que se avaliaram incapazes em virtude da AIDS apresentou escores bem inferiores ao grupo dos indivíduos sem incapacidade.

$\mathrm{O}$ presente estudo obteve excelentes resultados para a consistência interna, com alfas de Cronbach sempre acima do valor de corte $(0,70)$. O domínio preocu- pações com a medicação foi o que apresentou valor mais baixo $(0,73)$. Esse domínio também apresentou valor mais baixo $(0,57)$ em um dos trabalhos do autor do HAT-QoL (14). Provavelmente, esse valor foi afetado por 31 pacientes que não estavam tomando medicação no período da pesquisa e que não responderam esses itens.

Em relação a trabalhos anteriores sobre o HAT-QoL, adicionamos a este estudo a avaliação da confiabilidade intra e interobservador, que não tinham sido avaliadas até então. Ao contrário do esperado, os valores dos CCI foram superiores na confiabilidade interobservador $(0,87$ a 0,98$)$ do que na confiabilidade intraobservador $(0,82$ a 0,97$)$, provavelmente pelo período de tempo decorrido entre as aplicações. Na confiabilidade interobservador, o período entre as aplicações foi de 30 a 60 minutos no máximo, enquanto que na confiabilidade intraobservador o intervalo foi de até 15 dias. Isso sinaliza que talvez o período de aplicação do instrumento não deva ultrapassar 15 dias, com perigo de comprometimento das respostas pelo viés de memória.

O presente estudo apresentou algumas limitações. O tamanho da amostra $(n=$ 106) e o fato de ela não ter sido aleatória podem ter introduzido um viés. Talvez os indivíduos que aceitaram participar da pesquisa estivessem melhor fisicamente, aceitassem melhor a doença e não se importassem de responder perguntas sobre o assunto. Além disso, devido à dimensão continental do país, sua diversidade, e às especificidades sociais, econômicas e culturais de cada região, essa amostra talvez não seja representativa da parcela populacional que vive com HIV / AIDS no Brasil. Por tratar-se de um estudo preliminar, os resultados devem ser interpretados com cautela.

\section{CONCLUSÃO}

A versão em português do Brasil do HAT-QoL se mostrou compreensível e de fácil aplicação, comprovando o sucesso da adaptação cultural. Os resultados preliminares sugerem que o instrumento possui boa validade de construto e excelente confiabilidade. O questionário poderá contribuir, em certa medida, para a avaliação da qualidade de vida na população com HIV/AIDS no Brasil. O uso do questionário em pesquisas futuras também poderá contribuir para o aumento da 
compreensão do impacto de tratamentos na melhora da função de áreas consideradas importantes pelos pacientes. É pre-

1. Palella FG Jr, Delaney KM, Moorman AC, Loveless MO, Fuhrer J, Satten GA, et al. Declining morbidity and mortality among patients with advanced human immunodeficiency virus infection. N Engl J Med. 1998;338(13):853-60.

2. Detels R, Munoz A, McFarlane G, Kingsley LA, Margolick JB, Giorgi J, et al. Effectiveness of potent antiretroviral therapy on time to AIDS and death in men with known HIV infection duration. Multicenter AIDS Cohort Study Investigators. JAMA. 1998;280(17):1497-503.

3. Gange SJ, Barron Y, Greenblatt RM, Anastos $\mathrm{K}$, Minkoff $\mathrm{H}$, Young M, et al. Effectiveness of highly active antiretroviral therapy among HIV-I infected women. J Epidemiol Community Health. 2002;56(2):153-9.

4. Coons SJ, Rao S, Keininger DL, Hays RD. A comparative review of generic quality-of-life instruments. Pharmacoeconomics. 2000;17(1): 13-35.

5. Wu AW, Revicki DA, Jacobson D, Malitz FE. Evidence for reliability, validity and usefulness of the Medical Outcomes Study HIV Health Survey (MOS-HIV). Qual Life Res. 1997;6(6):481-93.

6. Patient-reported outcome and quality of life instruments database (PROQOLID). Disponível em: http://www.proqolid.org/ proqolid/search_1/pathology_disease? $\mathrm{pty}=1929$. Acessado em 25 de outubro de 2007.

7. Lenderking WR, Testa MA, Katzenstein D, Hammer S. Measuring quality of life in early ciso que o HAT-QoL seja utilizado, para possibilitar uma avaliação longitudinal e para julgar a necessidade de refinamento dos domínios função sexual, aceitação do $\mathrm{HIV}$, confiança no profissional e preocupações com a medicação.

\section{REFERÊNCIAS}

HIV disease: the modular approach. Qual Life Res. 1997;6(6):515-30.

8. Kemppainen JK. Predictors of quality of life in AIDS patients. J Assoc Nurses AIDS Care. 2001;12(1):61-70.

9. Smith KW, Avis NE, Mayer KH, Swislow L. Use of the MQoL-HIV with symptomatic HIV-positive patients. Qual Life Res. 1997; 6(6):555-60.

10. Davis EA, Pathak DS. Psychometric evaluation of four HIV disease specific qualityof-life instruments. Ann Pharmacother. 2001; 35(5):546-52.

11. Clayson DJ, Wild DJ, Quaterman P, DupratLomon I, Kubin M, Coons SJ. A comparative review of health-related quality-of-life measures for use in HIV/AIDS clinical trials. Pharmacoeconomics. 2006;24(3):751-63.

12. Holmes WC, Shea JA. A new HIV/AIDS-targeted quality of life (HAT-QoL) instrument. Development, reliability, and validity. Med Care. 1998;36(2):138-54.

13. Holmes WC, Shea JA. Performance of a new, HIV/AIDS-targeted quality of life (HAT-QoL) instrument in asymptomatic seropositive individuals. Qual Life Res. 1997;6(6): 561-71.

14. Holmes WC, Shea JA. Two approaches to measuring quality of life in the HIV/AIDS population: HAT-QoL and MOS-HIV. Qual Life Res. 1999;8(6):515-27.

15. Wood-Dauphinee S. Assessing quality of life in clinical research: from where have we come and where are we going? J Clin Epidemiol. 1999;52(4):355-63.
16. Guillemin F, Bombardier C, Beaton D. Crosscultural adaptation of health-related quality of life measures: literature review and proposed guidelines. J Clin Epidemiol. 1993; 46(12): 1417-32.

17. Beaton DE, Bombardier C, Guillemin F, Ferraz MB. Guidelines for the process of crosscultural adaptation of self-report measures. Spine. 2000;25(24):3186-91.

18. Colton T. Statistics in medicine. 1st ed. Boston: Little, Brown and Company; 1974.

19. McHorney CA, Ware JE, Lu JFR, Sherbourne CD. The MOS 36-item short-form health survey (SF-36): III. Tests of data quality, scaling assumptions and reliability across diverse patient groups. Med Care. 1994;32(1): 40-66.

20. Cronbach LJ. Coefficient alpha and the internal structure of tests. Psychometrika. 1951; 16(3):297-334.

21. Galvão MT, Cerqueira AT, MarcondesMachado J. Avaliação da qualidade de vida de mulheres com HIV/AIDS através do HATQoL. Cad Saude Publica. 2004;20(2): 430-7.

22. Arpinelli F, Visona G, Bruno R, De Carli G, Apolone G. Health-related quality of life in asymptomatic patients with HIV. Evaluation of the SF-36 health survey in Italian patients. Pharmacoeconomics. 2000;18(1):63-72.

Manuscrito recebido em 11 de janeiro de 2008. Aceito em versão revisada em 2 de abril de 2008.
ABSTRACT

Brazilian-Portuguese translation and validation of the HIV/AIDS-Targeted Quality of Life Instrument

Key words
Objectives. To translate the HIV/AIDS-Targeted Quality of Life Instrument (HATQoL) into Brazilian Portuguese, culturally adapt it, and evaluate its psychometric properties (validity and reliability) as a Brazilian version.

Methods. This cross-sectional study was carried out at the laboratory of infectious diseases at Escola Paulista de Medicina (Universidade Federal de São Paulo). Data were collected on clinical and sociodemographic characteristics of $106 \mathrm{HIV}$-infected individuals who answered the HAT-QoL and the SF-36 ${ }^{\circledR}$. Pearson's correlation coefficient was used to measure construct validity. Reliability was assessed using Cronbach's alpha and intraclass correlation coefficients.

Results. The sample was $70.8 \%$ male. The mean age was 39.9 years, with $40.5 \%$ of the participants being homosexual or bisexual. Eleven $(10.4 \%)$ patients had a CD4 cell count $\leq 200$ cells $/ \mathrm{mm}^{3}$. A substantial ceiling effect was observed in 7 of 9 HAT-QoL domains (overall function, life satisfaction, health worries, medication worries, HIV acceptance, provider trust, and sexual function). Sexual function was the domain with the highest ceiling effect (63.2\%). A substantial floor effect (30.2\%) was observed for financial worries. Statistically significant associations were observed between the HAT-QoL domains and clinical and sociodemographic characteristics, as well as with SF-36 domains. Internal consistency was satisfactory (Cronbach's alpha $=0.73-0.90)$. Inter- and intraobserver reproducibility was very high (0.87-0.98 and $0.82-0.97$, respectively).

Conclusion. The Brazilian Portuguese version of the HAT-QoL is valid, reliable, and may contribute to evaluating the impact of HIV infection on the quality of life of patients in Brazil.

Reproducibility of results, validation studies, quality of life, Acquired Immunodeficiency Syndrome, Brazil. 